www. revistad yo. com

\title{
Confort adaptativo aplicado a edificios escolares y aplicabilidad en el sur de España
}

\author{
Pablo Aparicio-Ruiz, Luis Onieva, Alejandro Escudero-Santana, Jesús Muñuzuri
}

Recibido: 25 de Julio de 2018 / Aceptado: 8 de Octubre de 2018

\section{Resumen}

El objetivo de este estudio es analizar la aplicabilidad del confort adaptativo a edificios escolares en el sur de España. Se busca comprender mejor la percepción del confort térmico, encontrar las posibilidades de ahorro energético y analizar las características de los estudios en los escolares y sus aptitudes conductuales relacionadas. Además, se propone la metodología más idónea y los tamaños de las muestras de los estudios hasta ahora desarrollados, para definir el desarrollo de un estudio de confort adaptativo para edificios escolares en Andalucía, estos estudios están dirigidos a conseguir alcanzar el máximo confort posible junto al ahorro energético.

\section{Palabras clave}

Confort adaptativo; eficiencia energética; confort térmico; colegios; preferencia térmica.

\section{Introducción}

En el ámbito de la gestión de calidad, ecoeficiencia y sostenibilidad, encontramos uno de los objetivos marcados por Europa en la Directiva 2012/27/UE es la eficiencia energética, donde se imponen ahorros de energía en los edificios para reducir los consumos. En (Krawczyk, 2014) se muestra la alta dependencia existente entre la demanda y la ubicación, por esta razón en climas templados se consume menos energía para calefacción y mucho más para refrigeración. La ubicación de un edificio y sus características genera una dependencia de los sistemas de climatización cuando el clima es adverso. Los sistemas de climatización representan el 60$70 \%$ del uso total de energía en edificios no industriales donde la infiltración y la ventilación suponen entre el 30-50\% de este consumo de energía (Khan, 2008). Por ese motivo, las estrategias desarrolladas combinan el aumento de la estanqueidad de los edificios con la mejora de los sistemas de climatización y ventilación o HVAC (Heating, Ventilation and Air Conditioning).

Pablo Aparicio-Ruiz*
pabloaparicio@us.es
Luis Onieva*
onieva@us.es
Alejandro Escudero-Santana*
alejandroescudero@us.es
Jesús Muñuzuri*
munuzuri@us.es
*Departamento de Organización Industrial y Gestión de
Empresas II. Escuela Técnica Superior de Ingenieros. Uni-
versidad de Sevilla. Camino de los Descubrimientos s/n
41092

En España, el Real Decreto 486/1997 (por el que se establecen las disposiciones mínimas de seguridad y salud en los lugares de trabajo), se indica que la temperatura de los locales donde se realicen trabajos sedentarios propios de oficinas o similares estará comprendida entre 17 y $27^{\circ} \mathrm{C}$., mientras que aquellos espacios donde se realicen trabajos ligeros estarán comprendidos entre 14 y $25^{\circ} \mathrm{C}$. Sin embargo, en los edificios escolares se incumple las condiciones de confort.

En los ambientes escolares, la temperatura, por su relación con la actividad intelectual, y la calidad del aire interior (CAI) conforman dos de los parámetros de confort para mejorar el aprendizaje de los niños y mejorar los resultados académicos, lo cual genera presión en las normativas escolares de edificación.

Dos corrientes de análisis del confort son comúnmente aplicadas en los estudios, por una parte, el análisis del Voto Medio Estimado (VME) y el Porcentaje de Personas en Disconfort (PPD) basados en la ISO 7730, y los basados en el confort adaptativo, UNE-EN 15251.

El objetivo de este documento es revisar la literatura sobre el confort térmico en escolares, para analizar la aplicabilidad para desarrollar un proyecto dirigido a conseguir alcanzar el máximo confort posible junto al ahorro energético. En primer lugar, se presenta una descripción general sobre el confort adaptativo. En segundo lugar, una revisión de estudios previos en escolares. En tercer lugar, un análisis de aplicabilidad y la presentación de las fases de la metodología para desarrollar una ley de confort para alcanzar un ahorro energético en estos espacios. Por último, se presentan las conclusiones. 


\section{Confort adaptativo}

El modelo adaptativo se fundamenta principalmente en tres aspectos interrelacionados: psicológicos (expectativa y habituación de confort en relación con el clima interior y exterior), de comportamiento o conductual (estrategias de restitución de la situación de confort como apertura de ventanas, uso de parasoles, ventiladores y puertas) y fisiológicos (aclimatación, ropa e ingesta alimenticia).

Nicol \& Humphreys (2002) determinaron los modelos de confort adaptativo (rangos de aceptabilidad térmica), relacionando la temperatura de confort con la temperatura media exterior. La definición de una temperatura de confort está basada en una regresión [1].

$$
\mathrm{Tc}=\mathrm{m} \bullet \operatorname{Trm}+\mathrm{c}
$$

Donde la temperatura media exterior (Trm) representa el efecto de la climatología de los días previos (Tod-1), y se expresa mediante el alisamiento exponencial [2].

$$
\operatorname{Trm}=\alpha \bullet \operatorname{Trm}-1+(1-\alpha) \operatorname{Tod}-1
$$

Para alcanzar dicha ecuación, que define el comportamiento de la temperatura de confort en el interior del edificio, se realizan encuestas, de las cuales se extrae la sensación térmica de los sujetos expresada en forma de voto de sensación térmica (VST) en una escala de 7 puntos de ASHRAE (caliente, cálido, ligeramente cálido, neutro, un poco frío, fresco y frío). Griffiths (1990) propuso un único valor estándar (G), para predecir la temperatura de confort, Tconfort, a partir de la sensación térmica media o individual del VST y la temperatura del globo, Tglobo, usando la ecuación definida en [3].

$$
\text { Tconfort }=\text { Tglobo }-(\text { VST } / G)
$$

Con la ecuación [2] y [3] se construye el conjunto de datos del cual se obtienen los valores de m y c de la ecuación [1].

Muchos proyectos experimentales se han llevado a cabo en edificios con ventilación natural (VN), ya sea en entornos residenciales, universitarios, escolares o de oficina. En los edificios en modo híbrido o mixto se realiza una combinación de ventilación natural ( $\mathrm{VN}$ ) donde se aplican ventanas operables controladas manual o automáticamente o respiraderos de entrada pasivos, y sistemas de climatización (SA) mecánicos que proporcionan distribución de aire y alguna forma de enfriamiento. Estos edificios permiten que los espacios se ventilen naturalmente cuando es factible o deseable, y usan SA para enfriamiento adicional cuando la VN no es suficiente. Este modo de operación permite minimizar el uso significativo de energía y los costes de operación del aire acondicionado.

La primera diferencia entre los sistemas convencionales y los sistemas de modo mixto es que estos últimos tienen cierta inteligencia asociada para cambiar de un modo a otro (de $\mathrm{VN}$ a SA y viceversa), permitiendo la reducción del consumo de energía manteniendo la temperatura y el confort de sus ocupantes.

El modelo adaptativo de confort térmico en edificios de modo mixto ha sido verificado por diferentes estudios, si bien cabe destacar la aplicabilidad en edificios de oficina para el sur de España (Barbadilla-Martín, 2017). El número de estudios llevados a cabo en edificios híbridos es menor en comparación con los estudios de campo realizados en edificios $\mathrm{VN}$

\section{Estudios previos de confort en edificios educativos}

Pocos estudios previos de confort térmico con niños han realizado ajustes en el modelo VME con el fin de abordar la diferencia en las tasas metabólicas. Sin embargo, Havenith (2007) determinó que las tasas metabólicas para las actividades escolares se encuentran dentro de un rango de 52$64 \mathrm{~W} / \mathrm{m} 2$, que es aproximadamente un $10 \%$ menor que el equivalente de adultos para actividades sedentarias como el trabajo de oficina (70W/m2) (Nicol \& Humphreys 2002). El confort térmico en las escuelas se ha desarrollado ampliamente en varios países para evaluar el confort térmico de los alumnos. Esta sección resume algunos trabajos de la literatura, cuyos autores se relacionan en la Tabla 1. 
Tabla 1 Resumen de trabajos consultados.

\begin{tabular}{|c|c|c|c|c|c|c|}
\hline Articulo & Tipología & Alumnos & Encuestas & Edad & Parámetros físicos & Centros \\
\hline Humphreys (1977) & $\mathrm{VN}+\mathrm{SA}$ & 641 & 10,000 & $7-9$ & TG-TA-HR-VA & 5 \\
\hline Corgnati et al. (2007) & $\mathrm{VN}+\mathrm{C}$ & 427 & - & & TG-TA-HR-VA & 5 \\
\hline Wigö (2008) & $\mathrm{VN}+\mathrm{SA}$ & 40 & - & $10-19$ & TG-TA-HR-VA & 1 \\
\hline Hussein \& Rahman (2009) & $\mathrm{VN}+\mathrm{SA}$ & - & - & & TG-TA-HR-VA & 2 \\
\hline Hwang et al. (2009) & $\mathrm{VN}$ & 1,614 & - & $11-17$ & TG-TA-HR-VA & 14 \\
\hline Mors et al. (2011) & $\mathrm{VN}$ & 79 & 1,657 & $9-11$ & TG-TA-HR-VA & 3 \\
\hline Teli et al. (2012) & $\mathrm{VN}$ & 230 & 1,314 & $7-11$ & TG-TA-HR-VA- $\mathrm{CO}_{2}$ & 2 \\
\hline d'Ambrosio et al. (2013) & $\mathrm{VN}$ & 4,000 & - & & TG-TA-HR-VA & 6 \\
\hline De Giuli et al. (2014) & $\mathrm{VN}+\mathrm{C}$ & 62 & - & $9-11$ & TG-TA-HR-VA & 1 \\
\hline Dias Pereira et al. (2014) & $\mathrm{VN}$ & 45 & - & & TG-TA-HR-VA- $\mathrm{CO}_{2}$ & 1 \\
\hline Montazami \& Nicol (2013) & $\mathrm{VN}$ & - & - & & TG-TA-HR-VA- $\mathrm{CO}_{2}$ & 18 \\
\hline De Dear et al. (2015) & $\mathrm{VN}+\mathrm{SA}$ & - & 2,850 & $10-18$ & TG-TA-HR-VA & 9 \\
\hline
\end{tabular}

${ }^{1}$ Los tipos de edificio son: con ventilación natural (VN), con calefacción ( $\mathrm{VN}+\mathrm{C}$ ) y sistemas acondicionados (VN+SA) Los parámetros físicos analizados son: temp. de globo (TG), temperatura del aire (TA), humedad relativa (HR), velocidad del aire (VA) y concentración de $\mathrm{CO}_{2}$.
Como se puede observar en la Tabla 1 en la que se resume un conjunto de trabajos consultados, se observa que existen trabajos de diversa tipología, y si bien, los estudios no coinciden en el número de encuestas, alumnos o centros a estudiar, si se observa una coincidencia en los parámetros físicos, donde es común el uso de temperatura del aire, humedad relativa, velocidad del aire, en algún caso se incluye la concentración de $\mathrm{CO} 2$ y de como se pudo ver en la ecuación [3], se requiere el uso del parámetro temperatura de globo, si bien existen diferencias en el tamaño de este sensor, se observa que los experimentos ilustran la viabilidad de utilizar un termómetro de globo negro con $40 \mathrm{~mm}$ de diámetro. (Aparicio, 2016).

De los estudios analizados en la Tabla 1, se observan algunas características comunes que no se han indicado explícitamente, como son:

- Los periodos de análisis en los estudios, suelen ser periodos cortos de estudio, generalmente, no más de 15 días por estación climática.

- La realización de encuestas a los estudiantes se suele realizar con cuestionarios en papel, ya que no es muy apropiado el análisis en aulas de ordenadores por las ganancias internas que suponen el uso de equipos.
- En el caso de las encuestas, se destaca, que se suelen desarrollar con un diseño especial en base a los alumnos y sus capacidades, si bien lo más habitual es analizar alumnos del entorno de los 10 años.

- Respecto al número de encuestas en el día, este factor suele ser bajo, del entorno de 1 o 2 al día.

- Respecto al cuestionario, se destaca que se suele incorporan una preguntas de adaptación en la vestimenta, en especialmente, sobre el uso del jersey o similar.

Humphreys (1977) analizó el confort escolar con una temperatura entre $17-23^{\circ} \mathrm{C}$ durante dos veranos (14 días) y no apreció diferencias entre sexos, se analizaron alumnos y profesores en el aspecto térmico y de vestimenta.

Corgnati et al. (2007) estudiaron las preferencias térmicas en la ciudad de Turín (Italia). Se analizó el PPD y se comparó con los votos subjetivos, los resultados mostraron que las personas aceptan los ambientes juzgados como neutros o cálidos (más neutrales). Se realizó un cuestionario de información general junto a información de confort termal, visual, acústico y calidad de aire interior. El estudio se amplió al confort adaptativo en (Corgnati, 2009). En este trabajo se encontró una tendencia a ambientes ligeramente cálidos en el período invernal y a ambientes neutros en la temporada 
templada. Esto sugirió una hipótesis interesante sobre la tendencia en función de la temporada de análisis.

Wigö (2008) realizó cuestionarios de calidad del aire, sensación y preferencia térmica, centrándose en la velocidad de aire y observó que cuando los estudiantes fueron sometidos a una velocidad de aire intermitente, durante la primavera y el otoño, los resultados indicaron que las variaciones en la velocidad del aire hacen que las personas perciban el aire como más frío y más reconfortante que cuando la velocidad del aire es constante.

Hussein \& Rahman (2009) realizaron un estudio de campo sobre las condiciones ambientales y el confort de los habitantes de Malasia, con una duración de tres días (poco significativa) junto a un cuestionario de respuestas subjetivas y de vestimenta. El estudio concluyó que los habitantes en el medio ambiente tropical tienen una mayor tolerancia al calor y pueden adaptarse al entorno al que están acostumbrados (80\% de aceptación).

Hwang et al. (2009) plantean el carácter universal de la norma ASHRAE 55 y analizan la divergencia en la aceptación del confort de los alumnos de Taiwán (clima cálido y húmedo) durante el semestre de otoño, se realizó un análisis mensual con dos encuestas para cada día de visita. Se realizó un análisis tipo Probit de respuestas de aceptación térmica de los estudiantes, obteniéndose las zonas de confort en 20.1$28.4^{\circ} \mathrm{C}(90 \%)$ y $17.6-30.0^{\circ} \mathrm{C}(80 \%$ de aceptación $)$, respectivamente. En este caso $1.7^{\circ} \mathrm{C}$ más bajo que el recomendado por ASHRAE.

Mors et al. (2011) analizaron escuelas de primaria en los Países Bajos. Se realizó un análisis de 24 días, cubriendo invierno, primavera y verano. Las encuestas analizaron sensación y aceptación térmica junto con la vestimenta. Se calculó el VME y se observó una diferencia entre la adaptación en la vestimenta entre ambos sexos, donde las mujeres tenían mejor adaptación. Se compararon los votos con los límites de las predicciones adaptativas y observaron que los niños prefieren temperaturas más bajas que las predichas por estos métodos.

Teli et al. (2012) desarrollaron un primer estudio tanto del VME como del modelo adaptativo en escuelas del sur de Inglaterra, con encuestas realizadas en 12 días cada trimestre, donde se preguntó sólo por la aceptación de la temperatura, y si usaban jersey. Se usaron pegatinas como recompensa por las encuestas. Los resultados sugirieron que los niños tienen una sensación térmica más cálida que los adultos y preferirían un entorno térmico interior con temperaturas más bajas en comparación con los adultos. Se concluyó que puede ser debido a la tasa metabólica/ $\mathrm{kg}$ de los niños y a la relación con el exterior (recreos y actividades al aire libre) junto a que los niños no siempre adaptan su ropa a su sensación térmica o la influencia de las características de sus entornos interiores. Los resultados indicaron que la temperatura de confort alcanzada a través del VME fue $4^{\circ} \mathrm{C}$ inferior a la obtenida por los cuestionarios y la obtenida por el modelo adaptativo fue $2^{\circ} \mathrm{C}$ menor. El estudio continuó en 2015 donde se investigó el confort adaptativo a partir de un estudio durante cuatro meses (abril-junio) (Teli, 2015).

d'Ambrosio et al. (2013) analizaron el VME para entornos escolares del sur de Italia. Se realizó una comparación entre el VME y el VST calculado por la investigación subjetiva. Se observa que existe un factor corrector del VME para entornos con ventilación natural.

De Giuli et al. (2014) se centran en un análisis de la calidad del ambiente interior (CAI) y confort visual. El estudio duró tres meses con alumnos de una escuela en Padua (Italia) y se centra en observar la posible correlación entre la respuesta de los alumnos y el VME.

Dias Pereira et al. (2014) estudiaron durante un año dos aulas en Beja (Portugal). Los estudiantes contestaron un cuestionario respecto a la vestimenta, la aceptación, sensación y preferencia térmica en base a los cuales se analizó el VME.

Montazami \& Nicol (2013) analizaron el confort térmico en Londres (Reino Unido). Analizaron las nuevas directrices inglesas de sobrecalentamiento, que indican que un edificio no debe estar más de 120 horas cuando la temperatura del aire en la clase se eleve a más de $28^{\circ} \mathrm{C}$ (frente a las 80 horas de la norma antigua) incluyendo dos restricciones más. La primera es que la diferencia de temperatura media entre interna y externa no debe exceder $\operatorname{los} 5^{\circ} \mathrm{C}$. La segunda que la temperatura del aire interno cuando el espacio está ocupado no debe exceder los $32^{\circ} \mathrm{C}$.

El experimento tomaba medidas internas cada 30 minutos. Los autores llegaron a la conclusión de que existe una mejor correlación entre la estimación de los docentes y el porcentaje de ocasiones en que la temperatura interior excede la temperatura de referencia utilizando el enfoque adaptativo del confort térmico en lugar de utilizar una temperatura fija.

De Dear et al. (2015) analizaron la preferencia térmica de alumnos australianos de primaria y secundaria, observando que la temperatura operativa interna de alrededor de $22,5^{\circ} \mathrm{C}$ era la temperatura neutra y preferida de los estudiantes, que generalmente es más fría de lo esperado para los adultos en las mismas condiciones ambientales térmicas.

\section{Análisis de aplicabilidad y propuesta de metodología}

La integración del confort adaptativo en la arquitectura a menudo requiere un estudio para evaluar los beneficios y la eficiencia. En la mayoría de los casos se requiere un buen conocimiento de las condiciones climáticas para tener éxito en esta tarea. Se propone el análisis de un edificio escolar en la ciudad de Sevilla, cuya climatología se representa en el gráfico psicrométrico de la Figura 1. 
Figura 1 Gráfico psicrométrico de la ciudad de Sevilla (España).

\author{
0. Confort \\ 1. Ganancias térmicas internas \\ 2. Uso de ventilación por los ocupantes \\ 3. Inercia térmica y ventilación nocturna \\ 4. Enfriamiento evaporativo
}

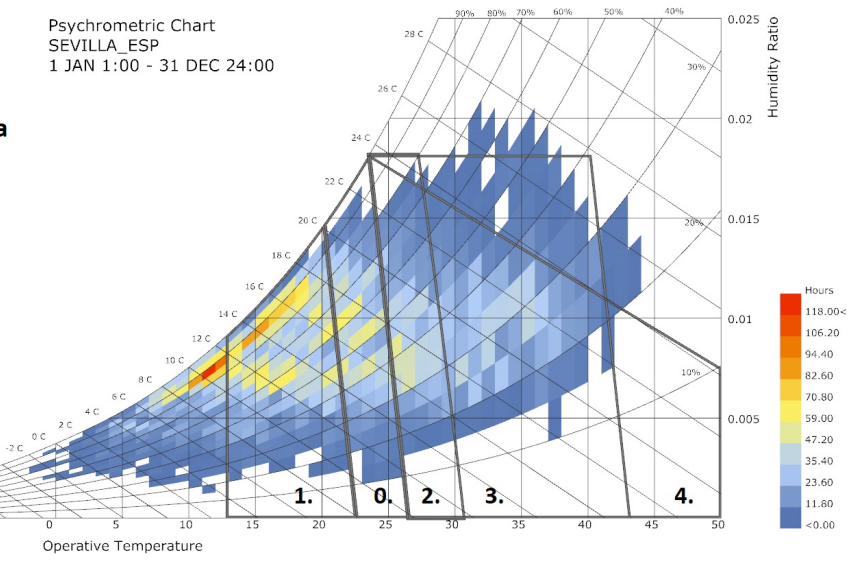

Una segunda fase de análisis de datos y desarrollo de la ley de confort del edificio en base a cuestionarios de los usuarios. Esta fase requiere de un estudio de los resultados de las encuestas. Al tratarse de estudiantes, se tendrá que descartar aquellas encuestas cuya respuesta no sea clara o aceptable. Además, la información de las encuestas debe de cruzarse con la información obtenida por los sensores, en base a la fecha y hora de respuesta de los cuestionarios por los alumnos.

La tercera fase corresponde a una segunda campaña experimental aplicando la ley de confort adaptativo, en esta fase, se podría experimentar con la activación de sistemas de climatización o ventilación de las aulas, en base a la temperatura de confort obtenida por la ley de control adaptativo; y finalmente, la cuarta fase se basa en el análisis de los ahorros asociados al edificio tras ambas campañas.

\section{Conclusiones}

La mayoría de los estudios realizan campañas con mediciones puntuales. Pocos estudios realizan un análisis de carácter anual con medidas periódicas de 10 a 30 min. y con encuestas diarias sobre el confort.

La aplicación del confort adaptativo en edificios está claramente justificada. En especial en climas con altas temperaturas. Igualmente existen pocos estudios de confort en colegios con $\mathrm{VN}+\mathrm{SA}$. La elección de la temperatura es fundamental, ya que alumnos y profesores, tienen distinta sensibilidad. Algunos estudios han analizado el papel de la etnia, el estado socioeconómico, la calidad del docente y el tamaño de la escuela en los logros del aprendizaje, áreas en las que se podría extender el estudio. Al igual que la influencia de la posición de los niños dentro del aula en términos de confort térmico, para ver si los que están sentados a lo largo de la pared externa y acristalada expresan un nivel de confort diferente con respecto a los que están sentados lejos de las ventanas. 
Es fundamental analizar la vestimenta para comparar las normativas ISO 7730 y EN15251. Se debe desarrollar un sistema que limite el exceso de temperatura siguiendo la normativa del Reino Unido.

Finalmente, se observa que, en el ámbito de los edificios escolares, se requieren sistemas de decisión para su aplicación en los sistemas activos, o sistemas que alerten de la necesidad de buscar medidas correctoras en la adaptación a la temperatura.

\section{Agradecimientos}

Los autores desean agradecer el apoyo financiero del proyecto DACAR (Ref. BIA2016-77431-C2-1-R) financiado por el Programa Estatal de Investigación, Desarrollo e Innovación Orientada a los Retos de la Sociedad (MINECO) y al proyecto ME4CA (Ref. P11-TEP-7247) financiado por la Consejería de Economía, Innovación, Ciencia y Empleo, Junta de Andalucía (Agencia de Innovación y Desarrollo de Andalucía).

\section{Referencias}

APARICIO, P.,SALMERÓN, J. M.,RUIZ, Á.,SÁNCHEZ, F. J.,AND BROTAS, L. (2016). «El termómetro de globo en estudios de confort y medioambiente en los edificios». Revista de La Construcción, 15(3), pp. 57-66.

BARBADILLA-MARTÍN， E.,SALMERÓN LISSÉN，J. M.,GUADIX MARTÍN, J.,APARICIO-RUIZ, P.,AND BROTAS, L. (2017). «Field study on adaptive thermal comfort in mixed mode office buildings in southwestern area of Spain». Building and Environment, 123. https:// doi.org/10.1016/j.buildenv.2017.06.042

CORGNATI, S. P.,ANSALDI, R.,AND FILIPPI, M. (2009). «Thermal comfort in Italian classrooms under free running conditions during mid seasons: Assessment through objective and subjective approaches». Building and Environment, 44(4), pp. 785-792. https://doi. org/10.1016/J.BUILDENV.2008.05.023

CORGNATI, S. P.,FILIPPI, M.,AND VIAZZO, S. (2007). «Perception of the thermal environment in high school and university classrooms: Subjective preferences and thermal comfort». Building and Environment, 42(2), pp. 951-959. https://doi.org/10.1016/j.buildenv.2005.10.027

D'AMBROSIO ALFANO, F. R.,IANNIELLO, E.,AND PALELLA, B. I. (2013). «PMV-PPD and acceptability in naturally ventilated schools». Building and Environment, 67, pp. 129-137. https://doi.org/10.1016/j.buildenv.2013.05.013
DE DEAR, R.,KIM, J.,CANDIDO, C.,AND DEUBLE, M. (2015). "Adaptive thermal comfort in australian school classrooms». Building Research and Information, 43(3), pp. 383-398. https://doi.org/10.1080/09613218.2015.99 1627

DE GIULI, V.,ZECCHIN, R.,CORAIN, L.,AND SALMASO, L. (2014). «Measured and perceived environmental comfort: Field monitoring in an Italian school». Applied Ergonomics, 45(4), pp. 1035-1047. https://doi. org/10.1016/j.apergo.2014.01.004

DIAS PEREIRA, L.,RAIMONDO, D.,CORGNATI, S. P.,AND GAMEIRO DA SILVA, M. (2014). «Assessment of indoor air quality and thermal comfort in Portuguese secondary classrooms: Methodology and results»». Building and Environment, 81, pp. 69-80. https://doi. org/10.1016/i.buildenv.2014.06.008

GRIFFITHS, I. (1990).«Thermal comfort studies in buildings with passive solar features : Field studies».Report to the Commission of the European Community, ENS35 090 UK.

HUMPHREYS, M. A. (1977). "A study of the thermal comfort of primary school children in summer». Building and Environment, 12(4), pp. 231-239. https://doi. org/10.1016/0360-1323(77)90025-7

HUSSEIN, I.,AND RAHMAN, M. H. A. (2009). «Field study on thermal comfort in Malaysia». European Journal of Scientific Research, 37(1), pp. 134-152.

HWANG, R.-L.,LIN, T.-P.,CHEN, C.-P.,AND KUO, N.-J. (2009). «Investigating the adaptive model of thermal comfort for naturally ventilated school buildings in Taiwan». International Journal of Biometeorology, 53(2), pp. 189-200. https://doi.org/10.1007/s00484-008-0203$\underline{2}$

KHAN, N.,SU, Y.,AND RIFFAT, S. B. (2008). «A review on wind driven ventilation techniques». Energy and Buildings, 40(8), pp. 1586-1604. https://doi.org/10.1016/j. enbuild.2008.02.015

KRAWCZYK, D. A. (2014). «Theoretical and real effect of the school's thermal modernization - A case study». Energy and Buildings, 81, pp. 30-37. https://doi. org/10.1016/j.enbuild.2014.04.058

MONTAZAMI, A.,AND NICOL, F. (2013). «Overheating in schools: Comparing existing and new guidelines».In Building Research and Information. 41, pp. 317-329. Routledge. https://doi.org/10.1080/09613218.2013.770 $\underline{716}$ 
MORS, S.,HENSEN, J. L. M.,LOOMANS, M. G. L. C.,AND BOERSTRA, A. C. (2011). «Adaptive thermal comfort in primary school classrooms: Creating and validating PMV-based comfort charts». Building and Environment, 46(12), pp. 2454-2461. https://doi. org/10.1016/j.buildenv.2011.05.025

NICOL, J. F.,AND HUMPHREYS, M. A. (2002). «Adaptive thermal comfort and sustainable thermal standards for buildings». In Energy and Buildings. 34, pp. 563-572. Elsevier. https://doi.org/10.1016/S03787788(02)00006-3

TELI, D.,JAMES, P. A. B.,AND JENTSCH, M. F. (2015). «Investigating the principal adaptive comfort relationships for young children». Building Research and Information, 43(3), pp. 371-382. https://doi.org/10.1080/096 $\underline{13218.2015 .998951}$
TELI, D.,JENTSCH, M. F.,AND JAMES, P. A. B. (2012). «Naturally ventilated classrooms: An assessment of existing comfort models for predicting the thermal sensation and preference of primary school children». Energy and Buildings, 53, pp. 166-182. https://doi. org/10.1016/J.ENBUILD.2012.06.022

WIGÖ, H. (2008). «Effects of intermittent air velocity on thermal and draught perception during transient temperature conditions». International Journal of Ventilation, 7(1), pp. 59-66. https://doi.org/10.1080/14733315.2008 .11683799 